\title{
Uncountably many topological models for ergodic transformations
}

\author{
S. GLASNER AND D. RUDOLPH \\ Tel Aviv University, Ramat Aviv, Israel; University of Maryland, College Park, \\ MD 20742, USA
}

(Received 12 January 1984)

\begin{abstract}
Given a topological process $(X, \mu, T)$ where $T$ is a homeomorphism of the compact metric space $X$ which preserves the probability measure $\mu$ and is ergodic, we show that there exists an uncountable family $\left\{\left(X_{i}, \mu_{i}, T_{i}\right)\right\}_{i \in I}$ of topological processes such that for every $i,\left(X_{i}, \mu_{i}, T_{i}\right)$ is measure-theoretically isomorphic to $(X, \mu, T)$ but for every $i \neq j,\left(X_{i}, \mu_{i}, T_{i}\right)$ and $\left(X_{j}, \mu_{j}, T_{j}\right)$ are not almost topologically conjugate.
\end{abstract}

1. Let us call a system $(X, \mu, T)$ where $T$ is a homeomorphism of the compact metric space $X$ which preserves the probability measure $\mu$ a topological process. We say that two such topological processes $(X, \mu, T)$ and $(Y, \nu, S)$ are almost topologically conjugate if there exist residual invariant Borel sets $X_{0} \subset X$ and $Y_{0} \subset Y$ with $\mu\left(X_{0}\right)=\nu\left(Y_{0}\right)=1$ and a continuous isomorphism $\left(X_{0}, \mu, T\right) \rightarrow^{\phi}\left(Y_{0}, \nu, S\right)$.

In [1] M. Denker and $M$. Keane asked the following question. Given an ergodic topological process $(X, \mu, T)$, is the almost topological isomorphism class of $(X, \mu, T)$ always strictly smaller than the metrical isomorphism class? We show in this note that in fact there are always uncountably many topological processes all metrically isomorphic such that any two are not almost topologically conjugate.

In the case where $(X, \mu, T)$ is a Bernoulli shift U. Fiebig in [2] shows that there are no less than $2^{x_{0}}$ non-almost topologically conjugate models. We wish to thank the Technical University of Delft and in particular Prof. M. Keane for giving us the opportunity to work on this problem while visiting Delft.

2. Let $(X, T, \mu)$ be an ergodic topological process. For a countable collection $F$ of functions in $L_{\infty}(X, \mu)$ we let al $(F)$ be the smallest uniformly closed $T$-invariant subalgebra of $L_{\infty}(X, \mu)$ containing $F$ and $C(X)$, be the subalgebra of continuous functions. Let $X_{F}$ be the Gelfand space of the $C^{*}$-algebra al $(F)$. Then on $X_{F}$ there is defined a homeomorphism, also denoted by $T$, induced by the action of $T$ on al $(F)$. Also the measure $\mu$ which acts on $L_{\infty}(X, \mu)$ as a bounded linear functional induces a probability measure $\mu_{F}$ on $X$ which is $T$-invariant.

Since al $(F)$ is a separable algebra $X_{F}$ is a metric space and we conclude that $\left(X_{F}, \mu_{F}, T\right)$ is a topological process. The elements of $X_{F}$ are the multiplicative functionals on al $(F)$ and each of these induces, by restriction, a multiplicative 
functional on $C(X)$ i.e. a point of $X$. This defines a continuous measure preserving homomorphism $\pi$ of $\left(X_{F}, \mu_{F}, T\right)$ onto $(X, \mu, T)$.

The algebra al $(F)$ is isometrically isomorphic to $C\left(X_{F}\right)$ and we have

$$
C(X) \subset \text { al }(F) \subset L_{\infty}(X, \mu) \subset L_{2}(X, \mu) .
$$

Since $C(X)$ is $L_{2}$-dense in $L_{2}(X, \mu)$ and $C\left(X_{F}\right)$ is $L_{2}$-dense in $L_{2}\left(X_{F}, \mu_{F}\right)$ it follows that $L_{2}\left(X_{F}, \mu_{F}\right)$ is canonically isomorphic to $L_{2}(X, \mu)$. We conclude that the processes $\left(X_{F}, \mu_{F}, T\right)$ and $(X, \mu, T)$ are measure-theoretically isomorphic via $\pi$. Note that if $\tilde{f}$ is the function in $C\left(X_{F}\right)$ which corresponds to $f \in F$ then on a set of measure 1 in $X_{F}, \tilde{f}=f \circ \pi$.

Our next goal is to find a condition on $\left(X_{F}, \mu_{F}, T\right)$ which will ensure that is not almost topologically conjugate with $(X, \mu, T)$. Suppose therefore that for some invariant residual Borel sets $X_{0} \subset X$ and $X_{F, 0} \subset X_{F}$ of full measure there exists a continuous isomorphism $\left(X_{0}, \mu, T\right) \rightarrow{ }^{\phi}\left(X_{F, 0}, \mu_{F}, T\right)$. Then clearly the map $E=\pi^{\circ} \phi$ is a measure-theoretical automorphism of $(X, \mu, T)$. Moreover since for $f \in F$,

$$
f \circ E=f \circ \pi \circ \phi=\tilde{f} \circ \phi
$$

on a set of full measure in $X$ and since both $\tilde{f}$ and $\phi$ are continuous we can conclude the following:

If $\left(X_{F}, \mu_{F}, T\right)$ is almost topologically conjugate to $(X, \mu, T)$ then there exists a measure theoretical automorphism $E$ of $(X, \mu, T)$ for which $f \circ E$ is continuous on a subset of full measure in $X,(f \in F)$.

Let us call a function in $L_{\infty}(X, \mu)$ essentially continuous if it is continuous on a subset of full measure. In the next section we shall show that there always exists an $f \in L_{\infty}(X, \mu)$ such that for every measure-theoretical automorphism $E$ of $(X, \mu, T)$, $f \circ E$ is not essentially continuous, thereby proving the existence of at least one topological process metrically but not almost topologically isomorphic to $(X, \mu, T)$. (Take $F=\{f\}$.)

Suppose now that in this manner one can obtain only countably many pairwise non-almost topologically conjugate topological processes $\left\{\left(X_{F_{i}}, \mu_{F_{i}}, T\right)\right\}_{i=1}^{\infty}$. Let

$$
A=\operatorname{al}\left(\bigcup_{i=1}^{\infty} F_{i}\right)
$$

be the smallest closed invariant subalgebra of $L_{\infty}(X, \mu)$ which contains $\bigcup F_{i}$ and $C(X)$. Then as in the case of al $(F)$ above, there exists a topological (metric) process $(Y, \nu, T)$ such that $C(Y)$ is isometrically isomorphic to $A$ and a continuous homomorphism $(Y, \nu, T) \rightarrow^{\psi}(X, \mu, T)$ which is a measure-theoretical isomorphism.

Moreover there is for each $i$ a continuous homomorphism $(Y, \nu, T) \rightarrow^{\psi_{i}}$ $\left(X_{F_{i}}, \mu_{F_{i}}, T\right)$ and all the $\psi_{i}$ 's are measure-theoretical isomorphisms.

Next we choose a function $g \in L_{\infty}(Y, \nu)$ such that for every measure-theoretical automorphism $E$ of $(Y, \nu, T), g \circ E$ is not essentially continuous. Let $\left(Y_{g}, \mu_{g}, T\right) \rightarrow^{\pi}$ $(Y, \mu, T)$ be the corresponding topological process and homomorphism. Suppose for some $i$ there exists an almost topological isomorphism $\phi: X_{F_{i}} \rightarrow Y_{g}$. If $\tilde{g} \in C\left(Y_{g}\right)$ 
is the function corresponding to $g$ then the function

$$
g \circ E=g \circ \pi \circ \phi \circ \psi_{i}=\tilde{g} \circ \phi \circ \psi_{i}
$$

where $E=\pi^{\circ} \phi \circ \psi_{i}$ is essentially continuous contradicting our choice of $g$. Thus $\left(Y_{\mathrm{g}}, \nu_{\mathrm{g}}, T\right)$ is not almost topologically conjugate to any of the topological processes $\left(X_{F_{i}}, \mu_{F_{i}}, T\right) i=1,2, \ldots$ However identifying $L_{\infty}(Y, \nu)$ with $L_{\infty}(X, \mu)$ we can consider $g$ to be an element of $L_{\infty}(X, \mu)$ and then obviously $\left(X_{G}, \mu_{G}, T\right)$, where $G=\{g\} \cup \bigcup_{i=1}^{\infty} F_{i}$, is topologically isomorphic to $\left(Y_{g}, \nu_{g}, T\right)$. This contradicts our assumption on the exhaustive nature of the family $\left\{\left(X_{F_{i}}, \mu_{F_{i}}, T\right)\right\}_{i=1}^{\infty}$. Our conclusion therefore is that the number of pairwise non-almost topologically conjugate processes of the form $\left(X_{F}, \mu_{F}, T\right)$ for countable $F \subset L_{\infty}(X, \mu)$, is uncountable.

3. In the proof of the following proposition we use an idea of D. Ornstein who proved a special case of it.

Proposition. There exists a function $f \in L_{\infty}(X, \mu)$ such that $f \circ E$ is not essentially continuous for every measure-theoretical automorphism $E$ of $(X, \mu, T)$.

We first prove the following:

LEMMA. Given a natural number $n$ there exists a measurable subset $B \subset X$ such that

$$
\mu\left(\left\{x \in B: n_{B}(x)=n\right\} \cup\left\{x \in B: n_{B}(x)=n+1\right\}\right)=\mu(B),
$$

where $n_{B}(x)$ is the smallest integer $j \geq 1$ with $T^{j} x \in B$.

Proof. Let $k \geq n(n+1)$ and write $k=s n+r, 0 \leq r<n$, then $s \geq n+1$ and $s=r+t$ for $t \geq 0$. Therefore we have for every $k \geq n(n+1)$ the representation $k=t_{k} n+r_{k}(n+1)$ with $t_{k}$ and $r_{k}$ positive integers.

By the Kakutani-Rokhlin lemma we can find a set $A$ for which almost every point of $A$ returns to $A$ after at least $n(n+1)$ times. Let

$$
A_{k}=\left\{x \in A: n_{A}(x)=k\right\}
$$

and put

$$
B=\bigcup_{k=n(n+1)}^{\infty}\left(\left(\bigcup_{j=0}^{r_{k}-1} T^{j(n+1)} A_{k}\right) \cup\left(\bigcup_{j=0}^{t_{k}-1} T^{r_{k}(n+1)+j n} A_{k}\right)\right)
$$

where $k=t_{k} n_{k}+r_{k}(n+1)$.

Proof of the proposition. Let $\left\{D_{j}\right\}_{j=1}^{\infty}$ be a basis for open sets on $X$. Choose a sequence of positive integers $k_{j}$ so that $\sum_{j=1}^{\infty} 1 /\left(k_{j}-1\right)<\frac{1}{2}$.

By Furstenberg's multiple recurrence theorem, [3], there exists for each $k_{j}$ an $n_{j}$ with

$$
\mu\left(D_{j} \cap T^{n_{j}} D_{j} \cap \cdots \cap T^{k_{j} n_{j}} D_{j}\right)>0 .
$$

Let $A_{0}$ be measurable with $\mu\left(A_{0}\right)=\frac{1}{2}$; we define inductively a sequence of sets $A_{j}$, $j=1,2, \ldots$ such that for every $j \geq 1$ and $l \leq j$,

$$
\mu\left(A_{j} \cap T^{n_{1}} A_{j} \cap \cdots \cap T^{k_{i} n_{l}} A_{j}\right)=0 .
$$

Suppose $A_{j-1}$ is defined; let $B$ be measurable with $n_{B}(x)$ between $n_{j}\left(k_{j}-1\right)$ and $n_{j} k_{j}$ for almost every $x$ in $B$. This can be done by the lemma (e.g. take $n=n_{j} k_{j}-1$ and 
$\left.n+1=n_{j} k_{j}\right)$. Writing

$$
A_{j}=A_{j-1} \backslash\left(A_{j-1} \cap \bigcup_{i=0}^{n_{j}-1} T^{i} B\right),
$$

it is clear that $\boldsymbol{A}_{j}$ satisfies (ii) for every $l \leq j$. Moreover since the measure of $B$ is at most $1 /\left(k_{j}-1\right) n_{j}$ we omit from $A_{j-1}$ a set of measure at most $n_{j} /\left(k_{j}-1\right) n_{j}=1 /\left(k_{j}-1\right)$. Thus for $A=\bigcap_{j=1}^{\infty} A_{j}$ we have $\mu(A) \geq \frac{1}{2}-\sum_{j=1}^{\infty} 1 /\left(k_{j}-1\right)>0$ and

$$
\mu\left(A \cap T^{n_{i}} A \cap \cdots \cap T^{n_{j} k_{j}} A\right)=0, \quad j=1,2, \ldots
$$

Now let $f=1_{A}$ and suppose that for some measure-theoretical automorphism $E$ of $(X, \mu, T), f \circ E$ is essentially continuous. Then in particular the set $\{x: f \circ E(x)=1\}$ must contain, up to measure zero, one of the basic sets $D_{j}$. Hence we have for some $j$, and up to sets of measure zero, $E D_{j} \subset A$. However $E$ is measure preserving and commutes with $T$ so that (i) and (iii) cannot hold together. This contradiction completes the proof.

The use of Furstenberg's theorem in the proof above, though convenient is not essential. One can use the ergodic theorem to obtain (i) with a sequence $l_{j, 1}, l_{j, 2}, \ldots, l_{j, k_{j}}$ which is sufficiently close to $n_{j}, \ldots, k_{j} n_{j}$ to make the argument work.

It would be interesting to find out whether the topological models we constructed can be made strictly ergodic, thereby proving the existence of uncountably many non-almost topologically conjugate, strictly ergodic, topological processes which are metrically isomorphic to a given ergodic topological process.

It would seem absurd to require the continuum hypothesis to conclude that there is a continuum of finitary isomorphism classes within each measurable isomorphism class but our method of proof clearly requires it. Surely a more constructive approach can be found, along the lines of U. Fiebig's work for Bernoulli schemes.

\section{REFERENCES}

[1] M. Denker \& M. Keane. Almost topological dynamical systems. Israel J. of Math. 34 (1979), 139-160.

[2] U. R. Fiebig. A return time invariant for finitary isomorphism. Ergod. Th. \& Dynam. Sys. 4 (1984), 225-231.

[3] H. Furstenberg. Ergodic behavior of diagonal measures and a theorem of Szemerédi on arithmetic progressions. J. d'Analyse Math. 31 (1977), 204-256. 